Research Paper

\title{
Naringenin Ameliorates Renovascular Hypertensive Renal Damage by Normalizing the Balance of Renin-Angiotensin System Components in Rats
}

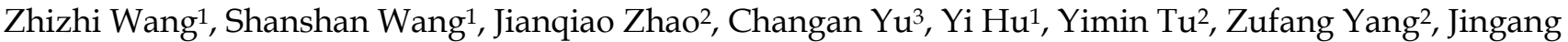 \\ Zheng1, 2, 4, Yong Wang1, ${ }^{\bowtie}$, Yanxiang Gao ${ }^{\star 凶}$ \\ 1. Department of Cardiology, China-Japan Friendship School of Clinical Medicine, Graduate School of Peking Union Medical College, Chinese Academy of \\ Medical Sciences, Beijing, 100029, China; \\ 2. Department of Cardiology, Peking University China-Japan Friendship School of Clinical Medicine, Beijing, 100029, China; \\ 3. Central Laboratory of Cardiovascular Disease, China-Japan Friendship Hospital, Beijing 100029, China; \\ 4. Department of Cardiology, China-Japan Friendship Hospital, Beijing 100029, China.
}

$\triangle$ Corresponding authors: Yanxiang Gao, PhD, Department of Cardiology, China-Japan Friendship Hospital, 2 Yinghua Dongjie, Chaoyang District, Beijing 100029, China. Phone: +86-10-84205625. Email: gaoyanxiang@zryhyy.com.cn or Yong Wang, MD, Department of Cardiology, China-Japan Friendship School of Clinical Medicine, Peking Union Medical College, Chinese Academy of Medical Sciences, Yinghua Dongjie, Chaoyang District, Beijing 100029, China. Phone/Fax: +86-10-64295131. E-mail: wangyong1239117@sina.com.

(C) Ivyspring International Publisher. This is an open access article distributed under the terms of the Creative Commons Attribution (CC BY-NC) license (https://creativecommons.org/licenses/by-nc/4.0/). See http://ivyspring.com/terms for full terms and conditions.

Received: 2018.10.30; Accepted: 2019.04.07; Published: 2019.05.07

\begin{abstract}
Background: Naringenin, a member of the dihydroflavone family, has been shown to have a protective function in multiple diseases. We previously demonstrated that naringenin played a protective role in hypertensive myocardial hypertrophy by decreasing angiotensin-converting enzyme (ACE) expression. The kidney is a primary target organ of hypertension. The present study tested the effect of naringenin on renovascular hypertensive kidney damage and explored the underlying mechanism.

Methods and Results: An animal model of renovascular hypertension was established by performing 2-kidney, 1-clip (2K1C) surgery in Sprague Dawley rats. Naringenin $(200 \mathrm{mg} / \mathrm{kg} / \mathrm{day})$ or vehicle was administered for 10 weeks. Blood pressure and urinary protein were continuously monitored. Plasma parameters, renal pathology and gene expression of nonclipped kidneys were evaluated by enzyme-linked immunosorbent assay, histology, immunohistochemistry, real-time polymerase chain reaction, and Western blot at the end of the study. Rats that underwent $2 \mathrm{~K} 1 \mathrm{C}$ surgery exhibited marked elevations of blood pressure and plasma Ang II levels and renal damage, including mesangial expansion, interstitial fibrosis, and arteriolar thickening in the nonclipped kidneys. Naringenin significantly ameliorated hypertensive nephropathy and retarded the rise of Ang II levels in peripheral blood but had no effect on blood pressure. $2 \mathrm{~K} 1 \mathrm{C}$ rats exhibited increases in the ACE/ACE2 protein ratio and ATIR/AT2R protein ratio in the nonclipped kidney compared with sham rats, and these increases were significantly suppressed by naringenin treatment.

Conclusions: Naringenin attenuated renal damage in a rat model of renovascular hypertension by normalizing the imbalance of renin-angiotensin system activation. Our results suggest a potential treatment strategy for hypertensive nephropathy.
\end{abstract}

Key words: naringenin, hypertensive nephropathy, renin-angiotensin system

\section{Introduction}

Hypertension is one of the most common diseases in the world. Based on a high blood pressure threshold of systolic blood pressure (SBP)/diastolic blood pressure $(\mathrm{DBP}) \geq 130 / 80 \mathrm{~mm} \mathrm{Hg}$, the crude prevalence of hypertension is $46 \%$ in adults aged 20 and older [1]. Every year, 9.4 million people die from complications of hypertension, and high blood pressure became the leading cause of death and disability-adjusted life worldwide in 2010 [2]. Among its complications, hypertensive nephropathy $(\mathrm{HN})$, 
also described as hypertensive glomerulosclerosis, is easily overlooked because of the lack of early clinical symptoms. Hypertensive nephropathy is one of the leading causes of end-stage renal disease (ESRD) in developed countries, second only to diabetic nephropathy [3]. Moreover, HN accounts for 34\% of incident ESRD cases in the United States population [4] and $23.4 \%$ of ESRD cases in Europe [5].

The pathophysiological mechanism of $\mathrm{HN}$ is complex, but the renin-angiotensin system (RAS) is known to play an important role. Inappropriate activation of the intrarenal local RAS contributes to the occurrence and development of hypertension and renal injury [6-8]. The current mainstay clinical therapy for $\mathrm{HN}$ involves targeting the RAS using angiotensin-converting enzyme (ACE) inhibitors (ACEIs) or angiotensin 1 receptor (AT1R) blockers (ARBs). These treatments protect the hypertensive kidney against proteinuria and impairments in renal function and retard the progression to ESRD [9-11]. However, a significant number of patients are still inadequately treated, partly because of side effects of these treatments, such as cough and angioedema. The other reasons for low treatment efficacy may involve compensatory RAS expression following inhibitor therapy via a feedback mechanism. Previous studies showed the increased plasma renin activity was associated with an increased risk of mortality in the patients of heart failure receiving RAS inhibitors [12], and renin expression was augmented in the fibrotic kidney by trandolapril [13].

Naringenin (4,5,7-trihydroxyflavanone) is a flavone compound that is enriched in citrus fruits. Accumulating evidence shows that naringenin has a pleiotropic protective function in many diseases, such as atherosclerosis, inflammatory bowel disease, diabetes mellitus, and cancer [14]. The main pharmacological effects of naringenin are antiinflammatory and antioxidant actions. Our recent study showed that naringenin inhibited left ventricular hypertrophy in rats that were subjected to $\mathrm{NG}^{\mathrm{G}}$-nitro-L-arginine methyl ester (L-NAME)-induced hypertension by downregulating the expression of $\mathrm{ACE}$ in the heart [15]. However, the effect of naringenin on hypertensive kidney injury has not been reported. The present study investigated the ways in which naringenin acts on hypertensive kidney damage and whether the RAS can be influenced by naringenin treatment.

\section{Materials and Methods}

\section{Animals and treatments}

Male Sprague Dawley rats, weighing 160-180 g, were obtained from Vital River Laboratory Animal Technology Co., Ltd (Beijing, China). The animals were housed in groups of 3-4 rats/cage in a well-ventilated room at $22^{\circ} \mathrm{C} \pm 3^{\circ} \mathrm{C}$ and $40-65 \%$ relative humidity under a $12 \mathrm{hr} / 12 \mathrm{hr}$ light/dark cycle. The rats were fed a normal diet and purified water.

After being matched for both body weight and $\mathrm{BP}$, the animals were randomized to the following groups ( $n=8 /$ group): sham operation + saline (Sham group), $2 \mathrm{~K} 1 \mathrm{C}+$ saline (2K1C group), and $2 \mathrm{~K} 1 \mathrm{C}+200$ $\mathrm{mg} / \mathrm{kg} /$ day naringenin (2K1C $+\mathrm{NGN}$ group). Naringenin was dissolved in a saline suspension. The rats were administered saline or naringenin daily by gavage, from 3 days before surgery until the end of the study. During this period, changes in body weight, heart rate, and BP were monitored.

The rats were sacrificed at the end of the 10th week of the study. They were anesthetized with 50 $\mathrm{mg} / \mathrm{kg}$ sodium pentobarbital, and arterial blood samples were obtained. After flushing with cold phosphate-buffered saline (PBS), the right (nonclipped) kidneys were quickly removed, weighed, and sliced. Both poles of each kidney were stored at $-80^{\circ} \mathrm{C}$ for the molecular analyses, the middle third part of kidney was fixed with $10 \%$ formalin for morphometric examinations.

All animal care and surgical procedures were approved by the China-Japan Friendship Hospital Animal Welfare and Ethics Committee (protocol no. 171001), which meets the United States National Institutes of Health guidelines for the care and use of laboratory animals.

\section{K1C surgical procedure}

Briefly, the 2K1C surgical procedure was performed as the following. The animals were anesthetized with $50 \mathrm{mg} / \mathrm{kg}$ sodium phenobarbital (i.p.) and placed in a prone position. A $3 \mathrm{~cm}$ long incision was made on the left beside the spine. The left kidney was carefully externalized and covered with wet gauze. For clipping, the renal artery of the left kidney was separated from the renal vein by blunt dissection. A silver clip ( $0.2 \mathrm{~mm}$ inner diameter) was placed around the renal artery, resulting in the partial occlusion of renal perfusion. The kidney was then gently pushed back into the retroperitoneal cavity, and the wound was closed layer by layer with sutures. In control rats, a sham surgical procedure was performed without clipping the artery.

\section{Non-invasive tail-cuff blood pressure measurement}

Blood pressure was recorded in all of the animals once daily for 3 days before $2 \mathrm{~K} 1 \mathrm{C}$ surgery to adapt the animals to tail-cuff plethysmography (Softron Biotechnology, Beijing, China). After surgery, 
blood pressure was monitored once weekly for the first 4 weeks and then once every 2 weeks. The BP and heart rate values are reported as an average of three consecutive measurements.

\section{Analysis of urinary albumin}

Every 2 weeks, the rats were placed in individual metabolic cages with free access to water for 24 hours to collect urine. The volume of urine was recorded. The urine was then centrifuged at 3000 rotations per minute (rpm) for $10 \mathrm{~min}$. The supernatant was separated and stored at $-80^{\circ} \mathrm{C}$ until the analysis. Urinary albumin concentrations were measured using an enzyme-linked immunosorbent assay (ELISA) kit (Bethyl Laboratories, Montgomery, Alabama, USA). Twenty four-hour albumin excretion was calculated by multiplying the urine albumin concentration by the urine volume, expressed as $\mu \mathrm{g} / 24 \mathrm{hr}$.

\section{Measurement of angiotensin II and Ang 1-7 concentrations in plasma}

The concentrations of angiotensin II (Ang II) and angiotensin 1-7 (Ang 1-7) in circulatory blood were measured using ELISA kits (Cusabio Biotech Co., Ltd, Wuhan, China). The ethylenediaminetetraacetic acid-anticoagulant arterial blood samples were centrifuged at $3000 \mathrm{rpm}$ for $10 \mathrm{~min}$ at $4^{\circ} \mathrm{C}$. The plasma supernatant was obtained and stored at $-80^{\circ} \mathrm{C}$. For the ELISA analysis, all of the samples were reconstituted and processed according to the manufacturer's recommendations.

\section{Histological analysis}

After dissection, the middle third part of the nonclipped kidney was placed in $10 \%$ formaldehyde. The tissues were paraffin-embedded and sliced into 3 $\mu \mathrm{m}$ sections. The sections were stained with hematoxylin-eosin (HE), Mason's trichrome, or Periodic acid-Schiff (PAS). Photographs were taken using a DP70 camera (Olympus America, Center Valley, PA, USA) at different magnifications. At least five randomly selected areas of each sample were photographed. According to the kidney chronicity grade [16], pathological scores were calculated as the sum of the following three items: (i) glomerulosclerosis $(0=<10 \%$ glomeruli with global and segmental sclerosis, $1=10-25 \%$ glomeruli with global and segmental sclerosis, $2=26-50 \%$ glomeruli with global and segmental sclerosis, $3=>50 \%$ glomeruli with global and segmental sclerosis), (ii) interstitial fibrosis $(0=<10 \%$ interstitial fibrosis, $1=10-25 \%$ interstitial fibrosis, $2=26-50 \%$ interstitial fibrosis, $3=$ $>50 \%$ interstitial fibrosis), (iii) arteriolar thickening (0 $=$ intimal thickening $<$ thickness of media, $1=$ intimal thickening $\geqq$ thickness of media). All histological analysis was done by a single investigator blinded to the grouping situation.

\section{Immunohistochemistry}

Paraffin-embedded sections $(3 \mu \mathrm{m})$ of nonclipped kidneys were deparaffinized, rehydrated, and heated by a microwave in citrate acid buffer $(\mathrm{pH}$ 6.0) to retrieve antigens. Endogenous peroxidase activity was blocked with $0.3 \%$ hydrogen peroxide. Antibodies specific for ACE, ACE2, AT1R, and AT2R (Abcam, Cambridge, MA, USA) were applied, and the slides were incubated overnight at $4^{\circ} \mathrm{C}$. On the second day, the sections were incubated with horseradish peroxidase-conjugated sheep anti-rabbit IgG (ZSGB$\mathrm{BIO}$, Beijing, China) at room temperature for $1 \mathrm{~h}$. Immunoreactivity was detected using a DAB substrate kit. Granular brown staining was considered positive. The nucleus was counterstained with Mayer's hematoxylin. For every sample, eight regions of the cortex and medulla, respectively, were examined under a BX53 microscope at 200× magnification (Olympus, Tokyo, Japan), photographed with a DP70 digital camera (Olympus America, Center Valley, PA, USA), and evaluated using ImageJ image analysis software (National Institutes of Health, Bethesda, MD, USA).

\section{Real-time quantitative polymerase chain reaction}

Total RNA from the cortex of the right (nonclipped) kidney was obtained by homogenization and isolation with Trizol (Applygen Technologies, Beijing, China). cDNA was reverse-transcribed from 1 $\mu \mathrm{g}$ of total RNA using a First Strand cDNA Synthesis kit (Promega, Madison, WI, USA). Real-time quantitative PCR was performed using TransStart Green qPCR Supermix (TransGen Biotech, Beijing, China). Rat-specific PCR primers for each gene were the following: AGT (forward, ACACCCCTGCTACA GTCCAC; reverse-TTTTCTGGGCAGCAAGAACT), renin (forward, TGGCAGATCACCATGAAGGG; reverse, TGCACAGGTCATCGTTCCTG), ACE (forward, CAGGGTCCAAGTTCCACGTT; reverse, GCC ACTGCTTACTGTAGCCCAA), ACE2 (forward, GAA TGCGACCATCAAGCG; reverse, CAAGCCCAGAG CCTACGA), AT1R (forward, GGAAACAGCTTGGT GGTGAT; reverse, CACACTGGCGTAGAGGTTGA), AT2R (forward, CAAACCGGCAGATAAGCATT; reverse, AAGTCAGCCACAGCCAGATT), and GAPDH (forward, GGTGAAGGTCGGTGTGAACG; reverse, TCCTGGAAGATGGTGATGGG). GAPDH was used as the reference gene. Polymerase chain reaction and analysis were performed using the Applied Biosystems ABI Prism 7500 system (Thermo Fisher Scientific, Waltham, MA, USA). 


\section{Western blot}

The nonclipped kidneys were sheared and homogenized in RIPA buffer (Sigma-Aldrich, St. Louis, MO, USA). The supernatant was obtained after centrifugation. Protein was quantified using bicinchoninic acid (BCA) method. A total of $80 \mu \mathrm{g}$ of protein of each sample was run using a sodium dodecyl sulfate-polyacrylamide gel electrophoresis (SDS-PAGE) system with stacking gel at $60 \mathrm{~V}$ and resolving gel at $120 \mathrm{~V}$. Protein was transferred to a nitrocellulose membrane at $250 \mathrm{~mA}$ at $4^{\circ} \mathrm{C}$ for $3 \mathrm{~h}$. The membrane was blocked with $5 \%(\mathrm{w} / \mathrm{v})$ nonfat dry milk in TBS-Tween for $1 \mathrm{~h}$ at room temperature, with slight rocking. The membrane was incubated with primary antibody in $5 \%(\mathrm{w} / \mathrm{v})$ nonfat dry milk in TBS-Tween overnight at $4^{\circ} \mathrm{C}$. After washing, the membrane was incubated with IRDye 700DXconjugated secondary antibody (Rockland, Gilbertsville, PA, USA) in TBS-Tween for $1 \mathrm{~h}$ at room temperature. Finally, the membrane was scanned using the Odyssey Infrared Imaging System (Li-Cor Biosciences, Lincoln, NE, USA). Final band intensities were dequantitated by normalizing to the GAPDH reference protein (anti-GAPDH antibody, CWBiotech, Beijing, China).

\section{Statistical analysis}

All of the continuous data are expressed as mean \pm SEM and were analyzed using GraphPad Prism 5.0 software (San Diego, CA, USA). For the statistical comparisons, the normality of the data was first evaluated. Equal variances were checked among normally distributed data, followed by one-way or two-way analysis of variance (ANOVA) for multiplegroup comparisons if the variances were equivalent. When the data were not normally distributed or when the variances were unequal, the nonparametric Kruskal-Wallis test followed by Dunn's post hoc test was used. Values of $p<0.05$ were considered statistically significant.

\section{Results}

\section{Effect of naringenin on renal physiopathology in $2 \mathrm{~K} 1 \mathrm{C}$ rats}

We established the rat model of renovascular hypertension by performing 2K1C surgery in Sprague Dawley rats. Beginning in week 2 after 2 K1C surgery, SBP began to increase and reached $163.2 \pm 15.3 \mathrm{mmHg}$ in the 2K1C group compared with $108.2 \pm 1.7 \mathrm{mmHg}$ in the sham group at 10 weeks (Fig. 1A). Urinary albumin excretion significantly increased from 6 weeks to 10 weeks after $2 \mathrm{~K} 1 \mathrm{C}$ surgery, which was slightly prevented by naringenin treatment (Fig. 1B, no statistical significance between $2 \mathrm{~K} 1 \mathrm{C}$ and $2 \mathrm{~K} 1 \mathrm{C}+$
NGN group). Sections of the right (nonclipped) kidney were histologically examined using $\mathrm{HE}$, Masson's trichrome, and PAS staining, which indicate arteriolar wall thickening, interstitial fibrosis, and glomerulosclerosis, in $2 \mathrm{~K} 1 \mathrm{C}$ rats compared with control rats. These parameters were forestalled by naringenin treatment (Fig. 1C). The pathological scores of the nonclipped kidneys were 0 in the sham group, $3.83 \pm 0.68$ in the $2 \mathrm{~K} 1 \mathrm{C}$ group $(p<0.001$, vs. sham group), and $1.40 \pm 0.35$ in the $2 \mathrm{~K} 1 \mathrm{C}+\mathrm{NGN}$ group ( $p<0.001$, vs. 2K1C group; Fig. 1D). Body weight and heart rate were not different among the three groups during the entire experimental period (Fig. S1A, B). These findings indicate a protective role for naringenin in $2 \mathrm{~K} 1 \mathrm{C}$-induced hypertensive renal injury.

\section{Effect of naringenin on circulating Ang II and Ang 1-7 levels in $2 \mathrm{~K} 1 \mathrm{C}$ rats}

To determine whether the circulating RAS participates in the pathogenesis of $2 \mathrm{~K} 1 \mathrm{C}$-induced hypertensive renal damage and the protective effect of naringenin, we measured the concentrations of Ang II and Ang 1-7 (i.e., the major physiologically active components of the RAS) in peripheral blood. Plasma Ang II levels in 2K1C rats were nearly two-fold higher than in sham rats $(13.27 \pm 1.78 \mathrm{pg} / \mathrm{ml}$ vs. $7.20 \pm 0.48$ $\mathrm{pg} / \mathrm{ml}, p<0.05)$. These elevations were significantly inhibited by naringenin treatment $(8.98 \pm 0.82 \mathrm{pg} / \mathrm{ml}$, $p<0.05$, vs. 2K1C group; Fig. 2A). Plasma Ang 1-7 levels were unaffected by $2 \mathrm{~K} 1 \mathrm{C}$ surgery or naringenin treatment (Fig. 2B).

\section{Effect of naringenin on local ACE/ACE2 expression in nonclipped kidneys in $2 \mathrm{~K} 1 \mathrm{C}$ rats}

Recent studies indicated that the intrarenal RAS plays an important role in the pathophysiology of hypertensive renal damage. We first examined the mRNA expression of integral components of the RAS in nonclipped kidneys using real-time PCR. As shown in Fig. 3, AT2R mRNA expression was downregulated, and the ACE/ACE2 transcriptional level ratio was upregulated in $2 \mathrm{~K} 1 \mathrm{C}$ rats. No effects on the expression of renin, angiotensinogen, ACE, ACE2, or AT1R were observed. Naringenin treatment upregulated AT2R mRNA expression but had no influence on the mRNA expression or the ratio of other components of the intrarenal RAS (Fig. 3).

We next examined the protein expression and distribution of components of the intrarenal RAS of nonclipped kidneys. Immunohistochemistry showed that ACE expression was unchanged in the cortex across the groups (Fig. 4A, B). Conversely, in the medulla, ACE expression was increased in $2 \mathrm{~K} 1 \mathrm{C}$ rats $(p<0.05)$, and this increase was prevented by 
naringenin treatment $(p<0.01$; Fig. 4 C, D). ACE2 expression in the cortex and medulla was downregulated in the $2 \mathrm{~K} 1 \mathrm{C}$ group $(p<0.01)$, and this change was significantly inhibited by naringenin treatment $(p<0.001$, Fig. 4A, B and C, D). The ratio of ACE/ACE2 immunostaining in both the cortex and medulla was significantly increased in $2 \mathrm{~K} 1 \mathrm{C}$ rats, and this increase was prevented by naringenin treatment (Fig. 4B, D). The Western blot results showed that ACE expression and the ACE/ACE2 ratio significantly increased in the $2 \mathrm{~K} 1 \mathrm{C}$ group, both of which were normalized by naringenin treatment (both $p<0.05$; Fig. 4E, F).

\section{Effect of naringenin on local AT1R/AT2R immunoreactivity in nonclipped kidneys in $2 \mathrm{~K} 1 \mathrm{C}$ rats}

The immunoreactivity of AT1R and AT2R in the renal cortex and medulla of nonclipped kidneys was also examined. As shown in Fig. 5, AT1R expression in the medulla tended to increase in the $2 \mathrm{~K} 1 \mathrm{C}$ group compared with the sham group, and this increase was

A

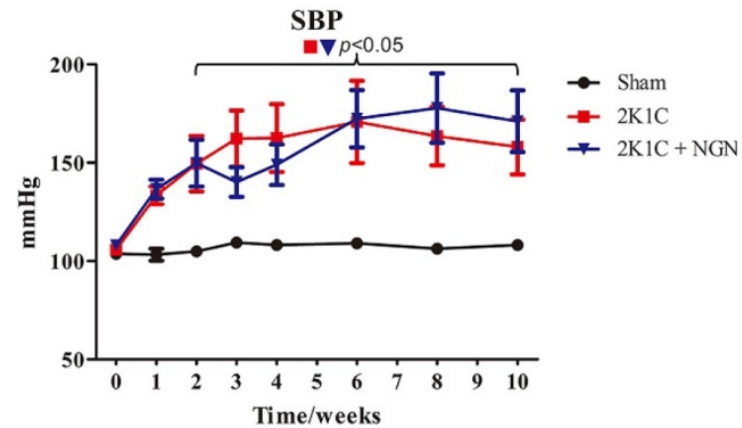

prevented by naringenin treatment $(p<0.01$; Fig. 5C, $D)$. Compared with control animals, AT2R expression decreased in the medulla in $2 \mathrm{~K} 1 \mathrm{C}$ rats $(p<0.01)$, and naringenin treatment prevented this decrease $(p<$ 0.05; Fig. 5C, D). The AT1R/AT2R ratio was calculated, and the results showed that AT1R/AT2R intensity significantly increased in the cortex and medulla in 2K1C hypertensive rats, and this increase was also prevented by naringenin treatment (Fig. 5B, D).

\section{Discussion}

The present study found that naringenin plays a protective role against hypertensive renal damage of nonclipped kidneys in $2 \mathrm{~K} 1 \mathrm{C}$ rats. In this process, systemic Ang II levels was suppressed and the balance of expression of local RAS components, including the ACE/ACE2 ratio and AT1R/AT2R ratio was preserved. This could highlight a potential mechanism for the beneficial effects of naringenin in hypertensive nephropathy.

B

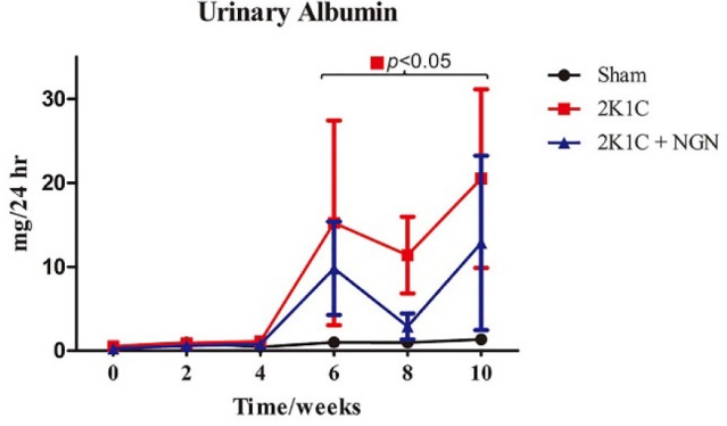

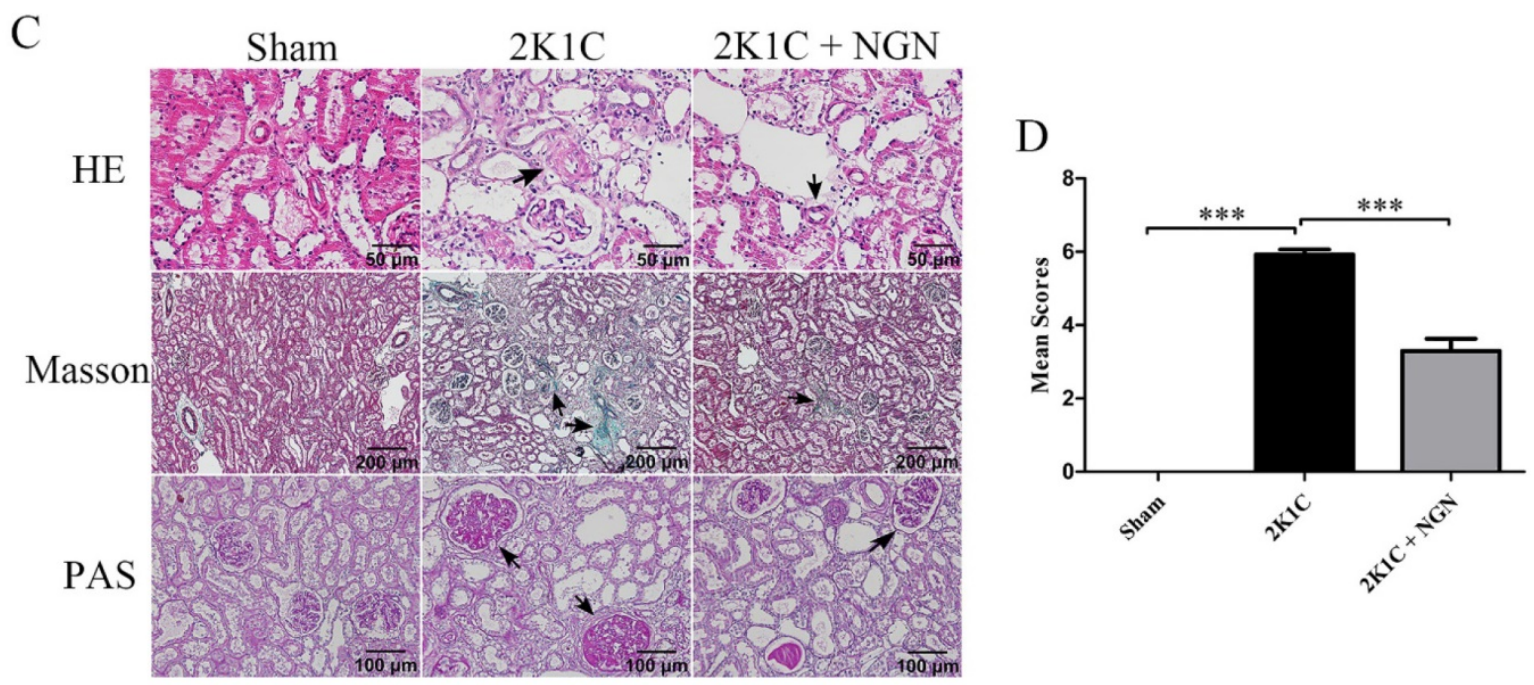

Figure 1. Comparison of blood pressure, urinary albumin, and renal histology in the sham, 2K1C, and 2K1C + NGN groups. (A) Systolic blood pressure (SBP), (B) Twenty four-hour urinary albumin excretion. Horizontal parentheses indicate significant differences versus the sham group. (C) Representative histopathological photographs of nonclipped kidneys stained with hematoxylin-eosin (400x), Masson trichrome (100x), and Periodic acid-Schiff (200x). Black triangles indicate arteriolar wall thickening, interstitial fibrosis, and glomerulosclerosis. (D) Pathological scores of the kidney. $n=8$ in each group. The data are expressed as mean \pm SEM. $* * * p<0.001$ 
A

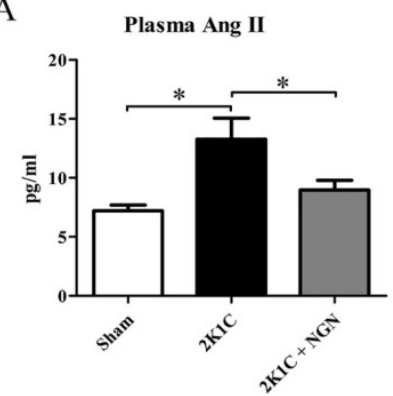

B

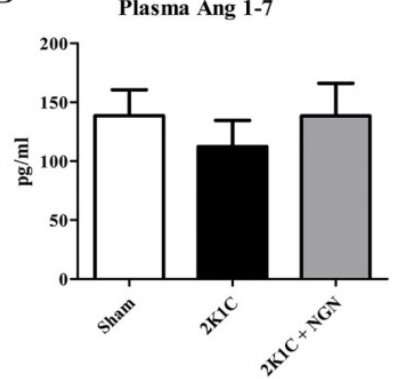

Figure 2. Effects of naringenin on circulating Ang II and Ang 1-7 levels in 2K1C rats. (A, B) Concentrations of Ang II (A) and Ang 1-7 (B) in plasma in the sham, $2 \mathrm{~K} 1 \mathrm{C}$, and $2 \mathrm{~K} 1 \mathrm{C}+\mathrm{NGN}$ groups. $\mathrm{n}=8$ in each group. The data are expressed as mean \pm SEM. $* p<0.05$.

A

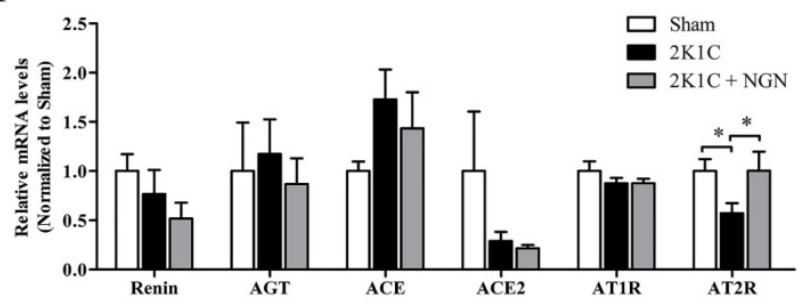

B

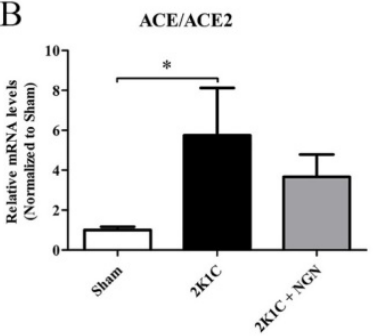

C

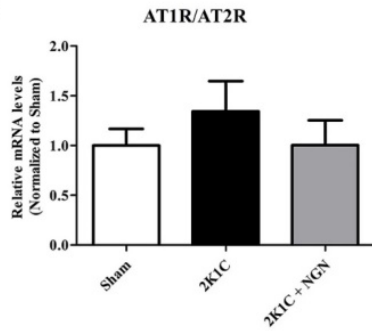

Figure 3. Effects of naringenin on the mRNA gene expression of RAS components in $2 \mathrm{~K} 1 \mathrm{C}$ rats. (A) Relative mRNA levels of renin, AGT, ACE, ACE2, ATIR, and AT2R in nonclipped kidneys, measured by quantitative PCR. (B) Ratio of ACE/ACE2 mRNA levels. (C) ATIR/AT2R mRNA levels. $n=8$ in each group. The data are expressed as mean \pm SEM. ${ }^{*} p<0.05$.

Naringenin is a natural flavonoid that is widely used in traditional Chinese medicine for its multiple pharmacological effects, including antiinflammatory and antioxidative actions. Previous studies reported that naringenin ameliorated diabetic nephropathy and obstructive renal fibrosis by inhibiting the transforming growth factor $\beta$ signaling pathway [17, 18]. It was also shown to attenuate nephrotoxicity by exerting antioxidative actions and suppressing AT1R and extracellular signal-regulated kinase 1/2-nuclear factor $\kappa B-m e d i a t e d$ inflammation $[19,20]$. However, the effect of naringenin on hypertensive renal damage was previously unknown. We established hypertensive animal model by $2 \mathrm{~K} 1 \mathrm{C}$ surgery in rats, and studied the effects of naringenin administration on hypertensive renal damage. According to the previous studies in renovascular hypertension model [21-23], the clipped kidney would present ischemic injury, and the hypertensive nephropathy was shown

in the nonclipped kidney, so we only studied nonclipped kidneys in our study. In the present study, nonclipped kidneys in $2 \mathrm{~K} 1 \mathrm{C}$ rats manifested typical hypertensive histopathology, including arteriolar alterations, glomerular damage, and tubulointerstitial changes, all of which were prevented by naringenin treatment.

In our rat model of 2K1C surgery-induced hypertension, BP increased after 2 weeks, and albuminuria developed after 6 weeks. The weight of the nonclipped kidneys increased at 10 weeks (Fig. S2). These parameters were unaffected by naringenin treatment. Similarly, our recent published data showed that naringenin improved left ventricular hypertrophy beyond lowering hypertension in rats that were subjected to L-NAME-induced hypertension [15]. Previous studies reported that treatment with naringenin or naringin, the glucoside of naringenin, improved hypertension in stroke-prone hypertensive rats and high-carbohydrate/high-fatdiet-fed obese rats [24, 25]. The various influences of naringenin on BP may be attributable to the use of different animal models.

Our results showed that the elevation of circulating Ang II levels in 2K1C rats was inhibited by naringenin treatment. Similarly, naringenin dosedependently reduced plasma Ang II levels in a rat model of cardiorenal syndrome [26]. The discordance we observed in the present study between BP and Ang II levels was consistent with a previous study that found that plasma Ang II levels were decreased by chymostatin treatment in a salt-dependent or Goldblatt-type rat model of hypertension, but chymostatin did not lower BP [27]. In addition, the elevation of circulating Ang II levels, regardless of an increase in renal perfusion pressure, was sufficient to induce kidney damage in hypertensive rats [28]. Besides the classical RAS, many alternative components in this system play critical roles in cardiorenal function. Ang 1-7 is recognized as a counterpart of Ang II that controls blood pressure in Ang II-dependent hypertension [29]. We also measured plasma concentrations of Ang 1-7. No significant changes were observed among the three groups, which is consistent with a previous study that found that plasma Ang1-7 levels did not decrease after 2K1C surgery [30].

Evidence indicates a role for the local RAS in damage to hypertension target organs [31, 32]. The ACE-Ang II-AT1 axis was regarded as the classic RAS pathway until the ACE2-Ang (1-7)-Mas axis was discovered in 2000 [33]. Subsequently, the latter axis was shown to counteract the effects of the former axis [34]. When the appropriate balance between ACE and ACE2 is disrupted, disease develops. Human kidney 
biopsies indicated that hypertensive patients have higher ACE/ACE2 mRNA ratios than normal controls [35]. Bernardi et al. reported a higher glomerular ACE/ACE2 ratio that was caused by the consumption of a high-salt diet in rats, resulting in oxidative stress and kidney damage [36]. Consistent with previous reports, the present study found that both the renal ACE/ACE2 mRNA ratio and ACE-ACE2 protein ratio in $2 \mathrm{~K} 1 \mathrm{C}$ hypertensive rats were significantly higher compared with sham rats [37]. Naringenin treatment normalized the disruption of the ACE/ACE2 protein ratio in both the cortex and medulla of the kidney.

AT2R was discovered in the 1980s [38]. Previous studies revealed that the role of the AT2R signaling pathway is opposite to the AT1R signaling pathway in diverse renal and cardiovascular pathologies. AT2R activation facilitates vasodilation and depresses inflammation and fibrosis in the heart, vascular wall, and kidney, independent of BP alterations [39]. In the present study, the AT1R/AT2R protein ratio was upregulated in both the cortex and medulla of the nonclipped kidney. This along with the imbalance of ACE/ACE2 could explain the kidney damage in $2 \mathrm{~K} 1 \mathrm{C}$ rats. The change in $\mathrm{AT} 1 \mathrm{R} / \mathrm{AT} 2 \mathrm{R}$ ratio was suppressed by naringenin treatment.

Pharmaceuticals that regulate RAS activity, especially ACEI and ARBs, are widely used to protect against hypertension-induced heart and kidney injury beyond reducing BP $[40,41]$. However, neither ACEI nor ARBs totally suppress the RAS, and combination therapy has failed to confer additional cardiovascular protection [41]. Dual RAS inhibition was associated with hypotension, hyperkalemia, and acute renal impairment, while lacking any beneficial effects on cardiovascular end points, such as death and myocardial infarction [42]. Treatment strategies that seek to suppress the imbalance of RAS component expression could be more effective for the treatment of HN. The present data showed that naringenin normalized the imbalance of the ACE/ACE2 and AT1R/AT2R protein ratios in the kidney in hypertensive rats, indicating that it might be a good treatment alternative for $\mathrm{HN}$.

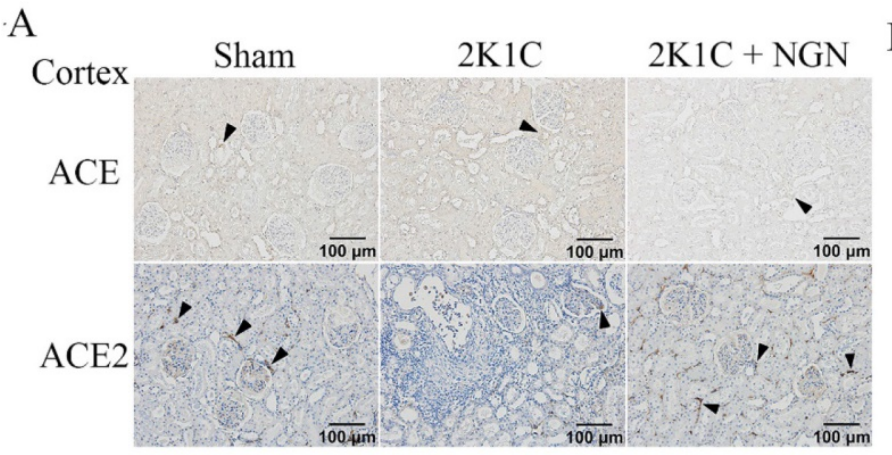

B

$\mathrm{C}$

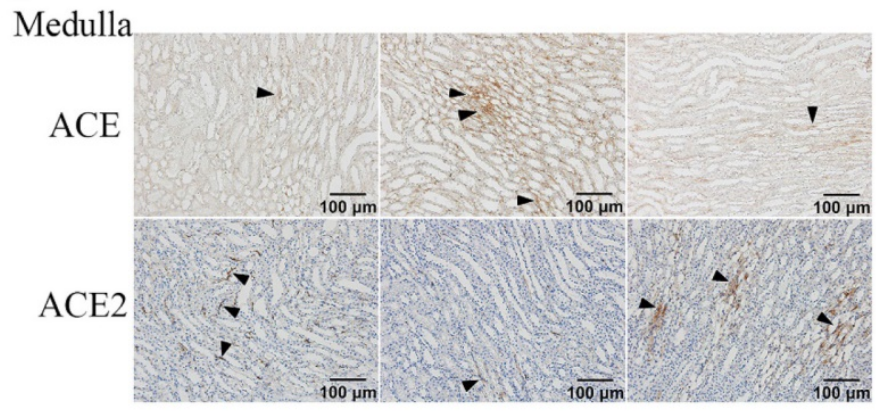

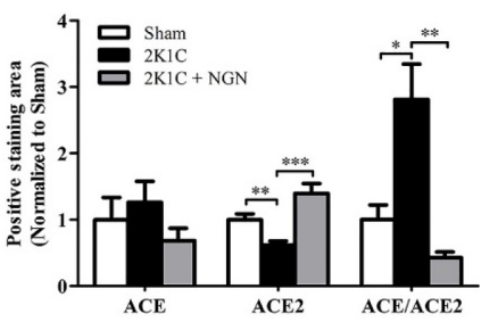

D

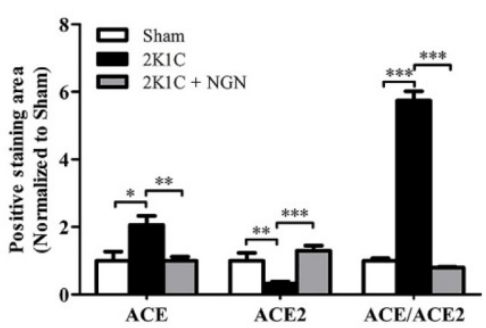

$\mathrm{E}$

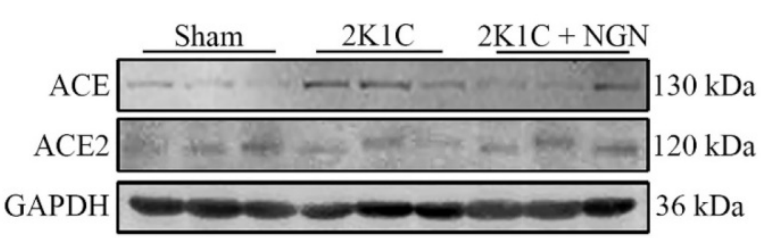

Figure 4. Effect of naringenin on $A C E$ and $A C E 2$ protein expression in nonclipped kidneys in $2 K 1 C$ rats. (A, B) Representative immunohistochemical staining of $A C E$ and ACE2 and quantification in the cortex. (C, D) Representative immunohistochemical staining of ACE and ACE2 and quantification in the medulla. (E, F) Representative Western blots of ACE and ACE2 in the kidney and quantification. Scale bars $=100 \mu \mathrm{m}$. Black triangles indicate representative positive staining. $\mathrm{n}=8$ in each group. The data are expressed as mean \pm SEM. $* p<0.05, * * p<0.01, * * * p 0.001$. 
A

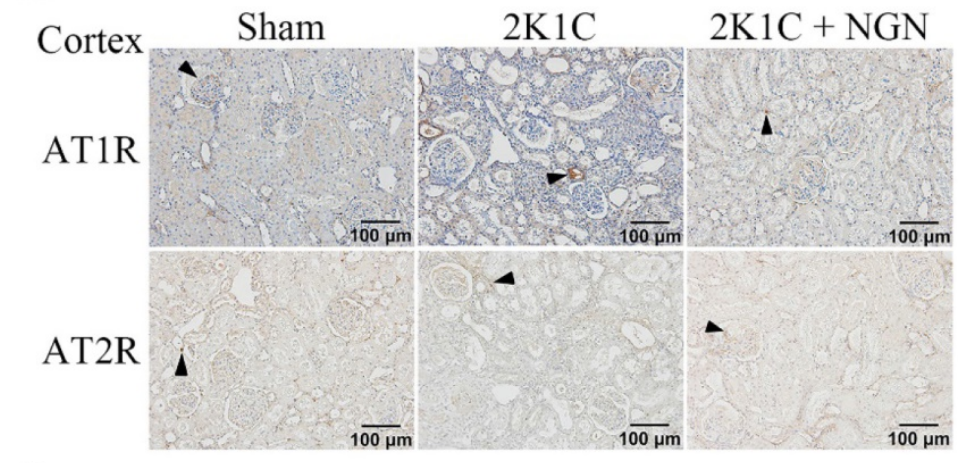

C

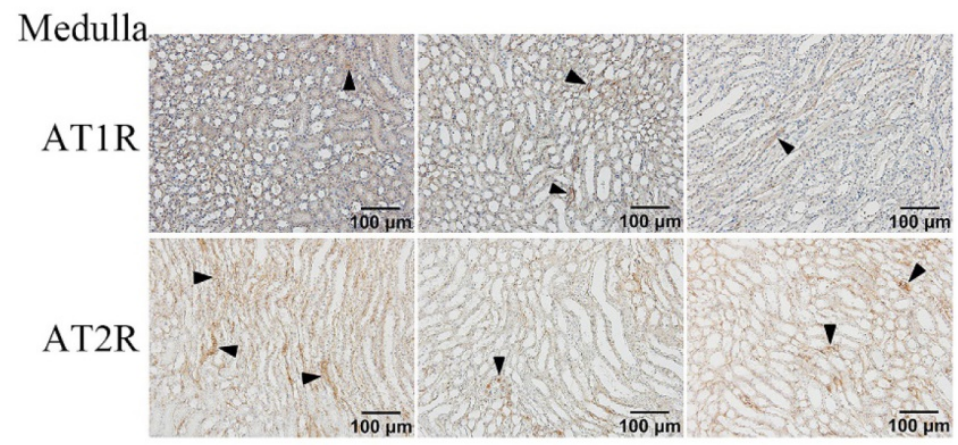

B

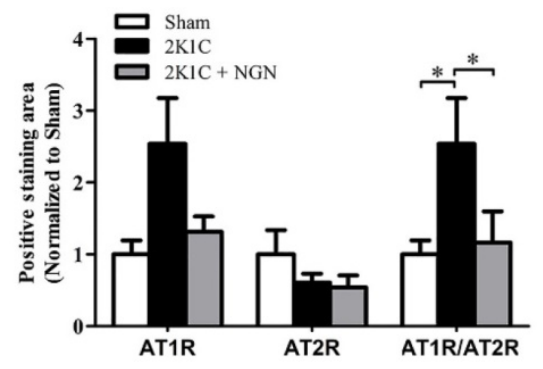

$\mathrm{D}$

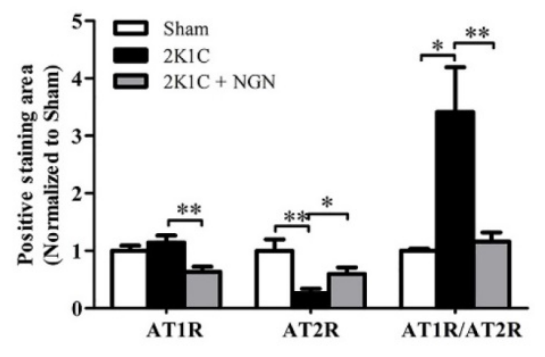

Figure 5. Effect of naringenin on ATIR and AT2R protein expression in nonclipped kidneys in $2 K 1 C$ rats. (A, B) Representative immunohistochemical staining of AT1R and AT2R and quantification in the cortex. $(C, D)$ Representative immunohistochemical staining of AT1R and AT2R and quantification in the medulla. Scale bars $=100 \mu \mathrm{m}$. Black triangles indicate representative positive staining. $\mathrm{n}=8$ in each group. The data are expressed as mean $\pm \mathrm{SEM}$. $* p<0.05, * * p<0.01$.

The most-reported effect of other flavonoids like quercetin, epigallocatechin-3-O-gallate, vaccarin and rutin in a similar model was antioxidant and anti-inflammatory [43-46], as well as inhibiting plasma renin, Ang II levels and ACE activities [45, 47], decreasing AT1R expression in myocardium or aorta $[45,47]$, and increasing cardiac AT2R expression [48]. But none of them have shown the ability of suppressing local ACE level in kidney as what naringenin did in our study.

The present study has several limitations. Based on prior evidence, we hypothesized that naringenin regulates the RAS by exerting antioxidative effects, reducing inflammation, or directly modulating the expression of RAS components. However, the precise mechanism of the effects of naringenin on the RAS needs further investigation. Besides, the clipped kidney produces the increase in angiotensin and renin in response to the reduced blood flow in $2 \mathrm{~K} 1 \mathrm{C}$ animal model, further studies are required to elucidate the effects of naringenin on the clipped kidney in relation to the abundance of components of the RAS.

In conclusion, the present findings suggest that naringenin may ameliorate hypertensive renal damage by modulating the balance of components of the RAS. Naringenin may be an effective therapy for hypertensive kidney disease.

\section{Abbreviations}

2K1C: 2-kidney, 1-clip; ACE: Angiotensinconverting enzyme; ACE2: Angiotensin-converting enzyme 2; Ang II: angiotensin II; Ang 1-7: angiotensin 1-7; AT1R: angiotensin II type 1 receptor; AT2R: angiotensin II type 2 receptor; ESRD: end-stage renal disease; HN: hypertensive nephropathy; NGN: naringenin; RAS: renin-angiotensin system.

\section{Supplementary Material}

Supplementary figures.

http://www.medsci.org/v16p0644s1.pdf

\section{Acknowledgements}

We thank Lin Pan, Jing Guo, Nannan Zhang, and Yan Wang for technical assistance.

\section{Funding}

This work was supported by the National Natural Science Foundation of China (81500326, 91639110) and Beijing Natural Science Foundation (7172195).

\section{Statement of Ethics}

Animal experiments conform to internationally accepted standards and have been approved by the China-Japan Friendship Hospital Animal Welfare and Ethics Committee (Protocol No. 171001). 


\section{Competing Interests}

The authors have declared that no competing interest exists.

\section{References}

1. Whelton PK, Carey RM, Aronow WS, Casey DE, Jr., Collins KJ, Dennison $\begin{array}{llll}\text { Himmelfarb } & \text { C, et } & \text { al. } & \end{array}$ ACC/AHA/AAPA/ABC/ACPM/AGS/APhA/

ASH/ASPC/NMA/PCNA Guideline for the Prevention, Detection, Evaluation, and Management of High Blood Pressure in Adults: Executive Summary: A Report of the American College of Cardiology/American Heart Association Task Force on Clinical Practice Guidelines. Hypertension. 2018; 71: 1269-324.

2. Lim SS, Vos T, Flaxman AD, Danaei G, Shibuya K, Adair-Rohani H, et al. A comparative risk assessment of burden of disease and injury attributable to 67 risk factors and risk factor clusters in 21 regions, 1990-2010: a systematic analysis for the Global Burden of Disease Study 2010. Lancet. 2012; 380: 2224-60.

3. Webster AC, Nagler EV, Morton RL, Masson P. Chronic Kidney Disease. Lancet. 2017; 389: 1238-52.

4. Saran R, Li Y, Robinson B, Ayanian J, Balkrishnan R, Bragg-Gresham J, et al. US Renal Data System 2014 Annual Data Report: Epidemiology of Kidney Disease in the United States. Am J Kidney Dis. 2015; 66: Svii, S1-305.

5. Kramer A, Pippias M, Noordzij M, Stel VS, Afentakis N, Ambuhl PM, et al. The European Renal Association - European Dialysis and Transplant Association (ERA-EDTA) Registry Annual Report 2015: a summary. Clin Kidney J. 2018; 11: 108-22.

6. Mennuni S, Rubattu S, Pierelli G, Tocci G, Fofi C, Volpe M. Hypertension and kidneys: unraveling complex molecular mechanisms underlying hypertensive renal damage. J Hum Hypertens. 2014; 28: 74-9.

7. Lee HA, Cho HM, Lee DY, Kim KC, Han HS, Kim IK. Tissue-specific upregulation of angiotensin-converting enzyme 1 in spontaneously hypertensive rats through histone code modifications. Hypertension. 2012; 59: 621-6.

8. Kobori H, Nangaku M, Navar LG, Nishiyama A. The intrarenal renin-angiotensin system: from physiology to the pathobiology of hypertension and kidney disease. Pharmacol Rev. 2007; 59: 251-87.

9. Zhou L, Liu Y. Wnt/ beta-catenin signaling and renin-angiotensin system in chronic kidney disease. Curr Opin Nephrol Hypertens. 2016; 25: 100-6.

10. Carey RM. The intrarenal renin-angiotensin system in hypertension. Adv Chronic Kidney Dis. 2015; 22: 204-10.

11. Ruggenenti P, Cravedi P, Remuzzi G. Mechanisms and treatment of CKD. J Am Soc Nephrol. 2012; 23: 1917-28.

12. Ueda T, Kawakami R, Nishida T, Onoue K, Soeda T, Okayama S, et al. Plasma renin activity is a strong and independent prognostic indicator in patients with acute decompensated heart failure treated with renin-angiotensin system inhibitors. Circ J. 2015; 79: 1307-14.

13. Tan $\mathrm{X}, \mathrm{He} \mathrm{W}$, Liu Y. Combination therapy with paricalcitol and trandolapril reduces renal fibrosis in obstructive nephropathy. Kidney Int. 2009; 76: 1248-57.

14. Rani N, Bharti S, Krishnamurthy B, Bhatia J, Sharma C, Kamal MA, et al. Pharmacological Properties and Therapeutic Potential of Naringenin: A Citrus Flavonoid of Pharmaceutical Promise. Curr Pharm Des. 2016; 22: 4341-59.

15. Gao $Y$, Wang $Z$, Zhang $Y$, Liu $Y$, Wang $S$, Sun $W$, et al. Naringenin inhibits $\mathrm{N}(\mathrm{G})$-nitro-L-arginine methyl ester-induced hypertensive left ventricular hypertrophy by decreasing angiotensin-converting enzyme 1 expression. Exp Ther Med. 2018; 16: 867-73.

16. Sethi S, D'Agati VD, Nast CC, Fogo AB, De Vriese AS, Markowitz GS, et al. A proposal for standardized grading of chronic changes in native kidney biopsy specimens. Kidney Int. 2017; 91: 787-9.

17. Yan N, Wen L, Peng R, Li H, Liu H, Peng H, et al. Naringenin Ameliorated Kidney Injury through Let-7a/TGFBR1 Signaling in Diabetic Nephropathy. J Diabetes Res. 2016; 2016: 8738760.

18. Meng XM, Zhang Y, Huang XR, Ren GL, Li J, Lan HY. Treatment of renal fibrosis by rebalancing TGF-beta/Smad signaling with the combination of asiatic acid and naringenin. Oncotarget. 2015; 6: 36984-97.

19. Karuppagounder V, Arumugam S, Thandavarayan RA, Pitchaimani V, Sreedhar R, Afrin R, et al. Naringenin ameliorates daunorubicin induced nephrotoxicity by mitigating AT1R, ERK1/2-NFkappaB p65 mediated inflammation. Int Immunopharmacol. 2015; 28: 154-9.

20. Fouad AA, Albuali WH, Zahran A, Gomaa W. Protective effect of naringenin against gentamicin-induced nephrotoxicity in rats. Environ Toxicol Pharmacol. 2014; 38: 420-9.
21. Vethe H, Finne K, Skogstrand T, Vaudel M, Vikse BE, Hultstrom M, et al. Distinct protein signature of hypertension-induced damage in the renal proteome of the two-kidney, one-clip rat model. J Hypertens. 2015; 33: 126-35.

22. Hartner A, Jagusch L, Cordasic N, Amann K, Veelken R, Jacobi J, et al. Impaired Neovascularization and Reduced Capillary Supply in the Malignant vs. Non-malignant Course of Experimental Renovascular Hypertension. Front Physiol. 2016; 7: 370.

23. Oboshi M, Naito Y, Sawada H, Iwasaku T, Okuhara Y, Eguchi A, et al. Attenuation of hypertension and renal damage in renovascular hypertensive rats by iron restriction. Hypertens Res. 2016; 39: 832-9.

24. Ikemura M, Sasaki Y, Giddings JC, Yamamoto J. Preventive effects of hesperidin, glucosyl hesperidin and naringin on hypertension and cerebral thrombosis in stroke-prone spontaneously hypertensive rats. Phytother Res. 2012; 26: 1272-7.

25. Alam MA, Kauter K, Brown L. Naringin improves diet-induced cardiovascular dysfunction and obesity in high carbohydrate, high fat diet-fed rats. Nutrients. 2013; 5: 637-50.

26. Liu Y, An W, Gao A. Protective effects of naringenin in cardiorenal syndrome. J Surg Res. 2016; 203: 416-23.

27. Roszkowska-Chojecka MM, Walkowska A, Gawrys O, Baranowska I, Kalisz M, Litwiniuk A, et al. Effects of chymostatin, a chymase inhibitor, on blood pressure, plasma and tissue angiotensin II, renal haemodynamics and renal excretion in two models of hypertension in the rat. Exp Physiol. 2015; 100: 1093-105.

28. Polichnowski AJ, Jin C, Yang C, Cowley AW, Jr. Role of renal perfusion pressure versus angiotensin II on renal oxidative stress in angiotensin II-induced hypertensive rats. Hypertension. 2010; 55: 1425-30.

29. Kuczeriszka M, Kompanowska-Jezierska E, Sadowski J, Prieto MC, Navar LG. Modulating Role of Ang1-7 in Control of Blood Pressure and Renal Function in AngII-infused Hypertensive Rats. Am J Hypertens. 2018; 31: 504-11.

30. Burgelova M, Vanourkova Z, Thumova M, Dvorak P, Opocensky M, Kramer HJ, et al. Impairment of the angiotensin-converting enzyme 2-angiotensin- (1-7)-Mas axis contributes to the acceleration of two-kidney, one-clip Goldblatt hypertension. J Hypertens. 2009; 27: 1988-2000.

31. Seccia TM, Caroccia B, Calo LA. Hypertensive nephropathy. Moving from classic to emerging pathogenetic mechanisms. J Hypertens. 2017; 35: 205-12.

32. Trimarco B, Santoro C, Pepe M, Galderisi M. The benefit of angiotensin AT1 receptor blockers for early treatment of hypertensive patients. Intern Emerg Med. 2017; 12: 1093-9.

33. Donoghue M, Hsieh F, Baronas E, Godbout K, Gosselin M, Stagliano N, et al. A novel angiotensin-converting enzyme-related carboxypeptidase (ACE2) converts angiotensin I to angiotensin 1-9. Circ Res. 2000; 87: E1-9.

34. Williams VR, Scholey JW. Angiotensin-converting enzyme 2 and renal disease. Curr Opin Nephrol Hypertens. 2018; 27: 35-41.

35. Wakahara S, Konoshita T, Mizuno S, Motomura M, Aoyama C, Makino $\mathrm{Y}$, et al. Synergistic expression of angiotensin-converting enzyme (ACE) and ACE2 in human renal tissue and confounding effects of hypertension on the ACE to ACE2 ratio. Endocrinology. 2007; 148: 2453-7.

36. Bernardi S, Toffoli B, Zennaro C, Tikellis C, Monticone S, Losurdo P, et al. High-salt diet increases glomerular ACE/ACE2 ratio leading to oxidative stress and kidney damage. Nephrol Dial Transplant. 2012; 27: 1793-800.

37. Prieto MC, Gonzalez-Villalobos RA, Botros FT, Martin VL, Pagan J, Satou R, et al. Reciprocal changes in renal ACE/ANG II and ACE2/ANG 1-7 are associated with enhanced collecting duct renin in Goldblatt hypertensive rats. Am J Physiol Renal Physiol. 2011; 300: F749-55.

38. Carey RM. Cardiovascular and renal regulation by the angiotensin type 2 receptor: the AT2 receptor comes of age. Hypertension. 2005; 45: 840-4.

39. Matavelli LC, Siragy HM. AT2 receptor activities and pathophysiological implications. J Cardiovasc Pharmacol. 2015; 65: 226-32.

40. Mukete BN, Cassidy M, Ferdinand KC, Le Jemtel TH. Long-Term Anti-Hypertensive Therapy and Stroke Prevention: A Meta-Analysis. Am J Cardiovasc Drugs. 2015; 15: 243-57.

41. Viazzi F, Bonino B, Cappadona F, Pontremoli R. Renin-angiotensinaldosterone system blockade in chronic kidney disease: current strategies and a look ahead. Intern Emerg Med. 2016; 11: 627-35.

42. Esteras R, Perez-Gomez MV, Rodriguez-Osorio L, Ortiz A, FernandezFernandez B. Combination use of medicines from two classes of reninangiotensin system blocking agents: risk of hyperkalemia, hypotension, and impaired renal function. Ther Adv Drug Saf. 2015; 6: 166-76.

43. Pereira SC, Parente JM, Belo VA, Mendes AS, Gonzaga NA, do Vale GT, et al. Quercetin decreases the activity of matrix metalloproteinase-2 and ameliorates vascular remodeling in renovascular hypertension. Atherosclerosis. 2018; 270: 146-53. 
44. Yi QY, Qi J, Yu XJ, Li HB, Zhang Y, Su Q, et al. Paraventricular Nucleus Infusion of Epigallocatechin-3-O-Gallate Improves Renovascular Hypertension. Cardiovasc Toxicol. 2016; 16: 276-85.

45. Zhu X, Zhou Z, Zhang Q, Cai W, Zhou Y, Sun H, et al. Vaccarin administration ameliorates hypertension and cardiovascular remodeling in renovascular hypertensive rats. J Cell Biochem. 2018; 119: 926-37.

46. Kaur S, Muthuraman A. Therapeutic evaluation of rutin in two-kidney one-clip model of renovascular hypertension in rat. Life Sci. 2016; 150: 89-94.

47. Wunpathe C, Potue P, Maneesai P, Bunbupha S, Prachaney P, Kukongviriyapan U, et al. Hesperidin Suppresses Renin-Angiotensin System Mediated NOX2 Over-Expression and Sympathoexcitation in 2K-1C Hypertensive Rats. Am J Chin Med. 2018; 46: 751-67.

48. Li J, Xie ZZ, Tang YB. Genistein prevents myocardial hypertrophy in 2-kidney 1-clip renal hypertensive rats by restoring eNOS pathway. Pharmacology. 2010; 86: 240-8. 